\title{
Effect of Temperature and Host Plant on the Biological Aspects of Tetranychus cucurbitacearum (Sayed) (Äcari: Tetranychidae)
}

\author{
M. F. Hassan ${ }^{*}$; A. F. El-Bahrawy ${ }^{* *}$; G. A. El-Kady ${ }^{* *}$; \\ Reham I. A. Abo-Shnaf ${ }^{* * *}$ and Marwa S. Kamel ${ }^{* *}$ \\ "Zoology and Agric. Nematology Dept., Fac. of Agric., Cairo Univ., Giza, Egypt \\ "*Plant Protec. Dept., Fac. of Agric., Suez Canal Univ., Ismailia, Egypt \\ ${ }^{* * *}$ Vegetable and Ornamental Acarology Dept., Plant Protec. Res. Instit., Agric. Res. Center, Dokki, Giza, Egypt
}

\begin{abstract}
The life history of the spider mite, Tetranychus cucurbitacearum (Sayed) was investigated on six eggplant cultivars ("White", "Black Stream beity", "Baladi", "Black king", "Roma" and "Taska") at 25 \& $35 \pm 2^{\circ} \mathrm{C}$ and $70 \pm 5 \%$ R.H. "Taska" cultivar shortened the life cycle to 10.08 and 6.93 days at 25 and $35^{\circ} \mathrm{C}$; while "White" cultivar prolonged this period to 16.28 and 10.61 days. The high egg production per female was on Taska cultivar ( $70.70 \& 47.58$ eggs) with a daily rate of $7.08 \& 5.50 \mathrm{egg} / \mathrm{female}$ at 25 and $35^{\circ} \mathrm{C}$, respectively. The low egg production per female was on White cultivar (41.66 \& 29.42 eggs) with a daily rate $7.38 \& 7.99 \mathrm{egg} /$ female, respectively at the same previous conditions.
\end{abstract}

Key Words: Biology, Spider mite, Reproduction, Eggplant, Cultivars.

\section{INTRODUCTION}

Eggplant, Solanum macrocarpon (L.) (Family: Solanaceae) is considered one of the most important vegetable crops. It is preferred by consumers and considered an economic crop that has a high rank locally. Also, it constitutes a huge part of exported crops to Arab and European markets. Eggplant is usually infested with several pests including the spider mites, Tetranychus urticae Koch and T. cucurbitacearum (Sayed) in Egypt (Farrag, 1975), T. cinnabarinus Boisduval, T. kanzawai Kishida, (Mansour \& Karachi, 1994 and Morishita \& Yano, 1996), and T. ludeni (Reedy 2001) in abroad. The spider mite, $T$. cucurbitacearum is a serious pest of a wide variety of economically important plants. It is confined to Delta and few areas in Upper Egypt (Farrag, 1975). Development from egg to adult often takes one to two weeks or more, depending on temperature, host plants humidity and other environmental factors (Zhang, 2003). Some studies on the biological aspects of this species on some different host plants were done by Hassan and Zaher, 1956; Abdel-Shaheed et al., 1971; Attiah et al., 1978 and Gomaa et al., 1987. This work aimed to study the effect of six eggplant cultivars on some biological aspects of $T$. cucurbitacearum. This knowledge may prove useful to our understanding of the population dynamics of $T$. cucurbitacearum for future programs of control management of this pest.

\section{MATERIALS AND METHODS}

Leaves of six eggplant cultivars: "White"; "Black Stream beity"; "Baladi"; "Black king"; "Roma" and "Taska" were used as substrates for rearing the spider mite, Tetranychus cucurbitacearum (Sayed) at two different temperatures: 25 and $35 \pm 2^{\circ} \mathrm{C}$ and constant R.H. $(70 \pm 5 \%)$. The stock culture started with one gravid female collected from eggplant cultivars in the farm of Faculty of Agriculture, Ismailia governorate. Eggplant leaf discs $(2 \mathrm{~cm}$ diameter) were placed on cotton bed in Phil dishes $(20 \mathrm{~cm} \times 15 \mathrm{~cm})$ with under surface upward. The cotton bed was kept wet by soaking with water twice daily so that the discs remained fresh. Newly deposited eggs of the same age were transferred singly, each to a leaf disc. Every dish contained 30 discs as replicates. Dishes with discs were kept in incubators with constant temperatures (25 and $35 \pm 2^{\circ} \mathrm{C}$ ) and $70 \pm 5 \%$ R.H. Discs of all treatments were examined twice daily and all biological aspects were recorded until death of mite adults.

\section{Statistical Analysis:}

The data were analyzed using the One Way ANOVAs complete randomized procedure with Duncan's HSD test at $\mathrm{P} \leq 0.5$ using the COSTAT 3.03system software (Quinn \& Keough, 2002).

\section{$\cdot$ RESULTS AND DISCUSSION}

Six different eggplant cultivars and two temperature degrees affected the duration of developmental stages as well as adult longevity and female fecundity of $T$. cucurbitacearum (Sayed) (Tables, $1 \& 2$ ).

Developmental rates of all mite stages are strongly influenced by temperatures. This fact has been well documented for many other tetranychid species (Laing, 1969; Hazen et al., 1973 and Carey \&Bradley, 1982). Total developmental times of 
Table (1): Effect of temperature on duration of T. cucurbitacearum reared on six eggplant cultivars.

\begin{tabular}{|c|c|c|c|c|c|c|c|}
\hline \multirow{3}{*}{ Stages } & \multirow{3}{*}{$\operatorname{sex}$} & \multicolumn{6}{|c|}{ Duration in days at two different temperatures and six cultivars } \\
\hline & & \multicolumn{2}{|c|}{ White } & \multicolumn{2}{|c|}{ Black Stream beity } & \multicolumn{2}{|c|}{ Baladi } \\
\hline & & $25^{\circ} \mathrm{C}$ & $35^{\circ} \mathrm{C}$ & $25^{\circ} \mathrm{C}$ & $35^{\circ} \mathrm{C}$ & $25^{\circ} \mathrm{C}$ & $35^{\circ} \mathrm{C}$ \\
\hline \multirow{2}{*}{ Egg } & 오 & $4.56 \pm 0.49 \mathrm{a}$ & $3.50 \pm 0.78 \mathrm{a}$ & $4.00 \pm 0.32 \mathrm{~b}$ & $2.60 \pm 0.63 b$ & $3.20 \pm 0.39 \mathrm{~cd}$ & $1.89 \pm 0.19 \mathrm{c}$ \\
\hline & $0^{\pi}$ & $4.21 \pm 0.33 \mathrm{a}$ & $3.08 \pm 0.49 a$ & $3.50 \pm 0.54 \mathrm{~b}$ & $2.62 \pm 0.37 \mathrm{~b}$ & $2.90 \pm 0.13 \mathrm{bc}$ & $1.54 \pm 0.46 \mathrm{c}$ \\
\hline \multirow{2}{*}{ Larva $\mathbf{A}$} & 우 & $2.30 \pm 0.53 \mathrm{ab}$ & $1.81 \pm 0.37 \mathrm{a}$ & $2.25 \pm 0.39 \mathrm{~b}$ & $1.78 \pm 0.42 \mathrm{a}$ & $1.94 \pm 0.24 \mathrm{c}$ & $0.89 \pm 0.17 \mathrm{~b}$ \\
\hline & $0^{\pi}$ & $2.08 \pm 0.20 \mathrm{a}$ & $0.83 \pm 0.25 \mathrm{a}$ & $1.58 \pm 0.40 \mathrm{bc}$ & $0.63 \pm 0.30 \mathrm{~b}$ & $1.75 \pm 0.30 b c$ & $0.33 \pm 0.20 \mathrm{c}$ \\
\hline \multirow[t]{2}{*}{ Q. } & 9 & $1.08 \pm 0.16 \mathrm{a}$ & $0.68 \pm 0.22 \mathrm{~b}$ & $0.82 \pm 0.15 \mathrm{~b}$ & $0.71 \pm 0.18 \mathrm{~b}$ & $0.59 \pm 0.16 \mathrm{c}$ & $0.46 \pm 0.14 \mathrm{c}$ \\
\hline & $\hat{0}$ & $0.71 \pm 0.10 \mathrm{a}$ & $0.42 \pm 0.10 \mathrm{ab}$ & $0.58 \pm 0.10 \mathrm{ab}$ & $0.46 \pm 0.10 \mathrm{bc}$ & $0.54 \pm 0.10 \mathrm{~b}$ & $0.33 \pm 0.10 \mathrm{ab}$ \\
\hline \multirow[t]{2}{*}{ Protonymph A } & $q$ & $2.88 \pm 0.20 \mathrm{a}$ & $1.92 \pm 0.19 \mathrm{a}$ & $2.27 \pm 0.27 \mathrm{~b}$ & $1.63 \pm 0.38 \mathrm{~b}$ & $1.92 \pm 0.16 \mathrm{c}$ & $1.17 \pm 0.19 \mathrm{c}$ \\
\hline & $\delta$ & $2.33 \pm 0.26 \mathrm{a}$ & $0.83 \pm 0.26 \mathrm{a}$ & $1.88 \pm 0.20 \mathrm{bc}$ & $0.66 \pm 0.30 \mathrm{bc}$ & $1.16 \pm 0.25 \mathrm{~d}$ & $0.50 \pm 0.00 \mathrm{c}$ \\
\hline Q. & $\frac{p}{9}$ & $1.13 \pm 0.20 \mathrm{a}$ & $0.58 \pm 0.12 \mathrm{~b}$ & $0.85 \pm 0.17 \mathrm{~b}$ & $0.89 \pm 0.19 \mathrm{a}$ & $0.80 \pm 0.10 \mathrm{bcd}$ & $0.60 \pm 0.13 b$ \\
\hline \multirow[b]{2}{*}{ Deutonymph A } & $\frac{0}{0}$ & $0.96 \pm 0.10 \mathrm{a}$ & $0.50 \pm 0.00 \mathrm{a}$ & $0.50 \pm 0.00 \mathrm{c}$ & $0.38 \pm 0.10 \mathrm{bc}$ & $0.58 \pm 0.12 \mathrm{c}$ & $0.33 \pm 0.13 \mathrm{c}$ \\
\hline & $\frac{f}{d}$ & $\frac{2.79 \pm 0.47 \mathrm{a}}{2.04 \pm 0.60 \mathrm{ab}}$ & $1.73 \pm 0.27 \mathrm{a}$ & $2.02 \pm 0.20 \mathrm{~cd}$ & $1.80 \pm 0.27 \mathrm{ab}$ & $1.79 \pm 0.18 \mathrm{~d}$ & $1.23 \pm 0.31 \mathrm{c}$ \\
\hline \multirow{2}{*}{ Q. } & $\frac{0}{9}$ & $\frac{2.04 \pm 0.60 \mathrm{ab}}{1.54 \pm 1.88 \mathrm{a}}$ & $1.16 \pm 0.20 \mathrm{~b}$ & $1.83 \pm 0.30 \mathrm{ab}$ & $0.71 \pm 0.33 \mathrm{c}$ & $1.54 \pm 0.40 \mathrm{ab}$ & $0.54 \pm 0.18 \mathrm{c}$ \\
\hline & $\frac{t}{3}$ & $\begin{array}{c}1.54 \pm 1.88 \mathrm{a} \\
0.79 \pm 0.10 \mathrm{a}\end{array}$ & $0.39 \pm 0.13 a$ & $1.33 \pm 0.30 \mathrm{ab}$ & $0.81 \pm 0.22 \mathrm{a}$ & $0.77 \pm 0.29 \mathrm{~b}$ & $0.73 \pm 0.17 \mathrm{a}$ \\
\hline \multirow[b]{2}{*}{ Total immatures } & $q$ & $\begin{array}{c}0.79 \pm 0.10 \mathrm{a} \\
11.72 \pm 2.23 \mathrm{a}\end{array}$ & $0.29 \pm 0.10 \mathrm{a}$ & $0.63 \pm 0.21 \mathrm{lb}$ & $0.83 \pm 0.49 a$ & $0.50 \pm 0.00 \mathrm{c}$ & $0.54 \pm 0.49 \mathrm{a}$ \\
\hline & $\frac{t}{\delta}$ & $\begin{array}{c}\frac{11.72 \pm 2.23 \mathrm{a}}{8.91 \pm 0.72 \mathrm{a}} \\
\end{array}$ & $7.11 \pm 0.49 \mathrm{ab}$ & $9.54 \pm 0.58 \mathrm{~b}$ & $7.62 \pm 0.90 \mathrm{a}$ & $7.81 \pm 0.43 \mathrm{c}$ & $5.08 \pm 0.62 \mathrm{c}$ \\
\hline \multirow[b]{2}{*}{ Life cycle } & ㅇ & $\begin{array}{c}8.91 \pm 0.72 \mathrm{a} \\
16.28 \pm 2.34 \mathrm{a}\end{array}$ & $4.03 \pm 0.68 \mathrm{a}$ & $7.00 \pm 0.32 \mathrm{~b}$ & $3.67 \pm 0.70 \mathrm{~b}$ & $6.07 \pm 0.40 \mathrm{~cd}$ & $2.57 \pm 0.52 \mathrm{c}$ \\
\hline & $0^{\pi}$ & $\frac{16.28 \pm 2.34 \mathrm{a}}{13.12 \pm 0.82 \mathrm{a}}$ & $10.61 \pm 0.80 \mathrm{a}$ & $13.54 \pm 0.62 \mathrm{~b}$ & $10.22 \pm 1.30 \mathrm{a}$ & $11.01 \pm 0.67 \mathrm{~d}$ & $6.97 \pm 0.69 \mathrm{~b}$ \\
\hline \multirow{2}{*}{ Adult longevity } & 오 & $\frac{13.12 \pm 0.82 \mathrm{a}}{8.79 \pm 0.59 \mathrm{e}}$ & $7.11 \pm 0.57 \mathrm{a}$ & $10.50 \pm 0.71 \mathrm{~b}$ & $6.29 \pm 0.86 \mathrm{~b}$ & $8.97 \pm 0.40 \mathrm{~cd}$ & $4.11 \pm 0.59 \mathrm{c}$ \\
\hline & $\hat{0}^{2}$ & $\begin{array}{l}8.79 \pm 0.59 \mathrm{e} \\
6.16 \pm 0.73 \mathrm{c}\end{array}$ & $6.78 \pm 0.58 \mathrm{e}$ & $13.42 \pm 0.74 \mathrm{~b}$ & $9.44 \pm 0.72 \mathrm{c}$ & $8.87 \pm 0.43 \mathrm{e}$ & $6.66 \pm 1.03 \mathrm{e}$ \\
\hline \multirow{3}{*}{ Life span } & 오 & & $5.13 \pm 0.34 \mathrm{~d}$ & $8.25 \pm 6.41 \mathrm{~b}$ & $5.90 \pm 0.73 \mathrm{bc}$ & $6.58 \pm 0.49 \mathrm{c}$ & $5.63 \pm 0.44 d$ \\
\hline & $0^{\pi}$ & $\frac{25.07 \pm 2.93 \mathrm{a}}{19.28 \pm 1.55 \mathrm{a}}$ & $\frac{17.39 \pm 1.384 a}{122440.91 a}$ & $26.96 \pm 1.36 \mathrm{c}$ & $19.66 \pm 2.02 \mathrm{~b}$ & $19.88 \pm 1.10 \mathrm{e}$ & $13.63 \pm 1.72 \mathrm{~d}$ \\
\hline & & & $12.24 \pm 0.91 \mathrm{a}$ & $18.75 \pm 7.12 b$ & $12.19 \pm 1.59 \mathrm{bc}$ & $15.55 \pm 0.89 \mathrm{~d}$ & $9.74 \pm 1.03 \mathrm{~d}$ \\
\hline \multirow{3}{*}{ Stages } & \multirow{3}{*}{$\operatorname{sex}$} & \multicolumn{6}{|c|}{ Duration in days at two different temperatures and six cultivars } \\
\hline & & \multicolumn{2}{|c|}{ Black king } & & & & \\
\hline & & $25^{\circ} \mathrm{C}$ & $35^{\circ} \mathrm{C}$ & $25^{\circ} \mathrm{C}$ & $25^{\circ} \mathrm{C}$ & $35^{\circ} \mathrm{C}$ & $25^{\circ} \mathrm{C}$ \\
\hline Egg & 오 & $4.56 \pm 0.49 \mathrm{a}$ & $3.18 \pm 0.35 \mathrm{a}$ & $3.35 \pm 0.43 \mathrm{c}$ & $2.75 \pm 0.50 \mathrm{~b}$ & $2.96 \pm 0.21 \mathrm{~d}$ & $2.02 \pm 0.44 c$ \\
\hline & 8 & $4.16 \pm 0.37 \mathrm{a}$ & $2.87 \pm 0.21 \mathrm{~b}$ & $2.90 \pm 0.20 b c$ & $2.83 \pm 0.25 \mathrm{~b}$ & $2.83 \pm 0.20 \mathrm{c}$ & $1.13 \pm 0.31 \mathrm{c}$ \\
\hline Larva A & $\frac{9}{1}$ & $2.54 \pm 0.49 \mathrm{a}$ & $1.66 \pm 0.26 \mathrm{a}$ & $2.14 \pm 0.29 \mathrm{~b}$ & $1.63 \pm 0.22 \mathrm{a}$ & $1.69 \pm 0.18 \mathrm{c}$ & $0.56 \pm 0.21 \mathrm{c}$ \\
\hline & $\frac{0}{9}$ & $\frac{1.95 \pm 0.10 \mathrm{ab}}{0.89 \pm 0.25 \mathrm{~b}}$ & $1.13 \pm 0.31 \mathrm{a}$ & $1.29 \pm 0.33 \mathrm{~d}$ & $0.54 \pm 0.10 \mathrm{bc}$ & $1.58 \pm 0.40 \mathrm{~cd}$ & $0.29 \pm 0.10 \mathrm{c}$ \\
\hline Q. & $\frac{7}{8}$ & $\frac{0.89 \pm 0.25 \mathrm{~b}}{0.58 \pm 0.10 \mathrm{ab}}$ & $0.87 \pm 0.16 \mathrm{a}$ & $0.77 \pm 0.16 \mathrm{~b}$ & $0.71 \pm 0.09 \mathrm{~b}$ & $0.54 \pm 0.09 \mathrm{c}$ & $0.47 \pm 0.07 \mathrm{c}$ \\
\hline & 9 & $\frac{0.58 \pm 0.10 \mathrm{ab}}{2.23 \pm 0.34 \mathrm{~b}}$ & $0.58 \pm 0.13 \mathrm{a}$ & $0.54 \pm 0.10 \mathrm{~b}$ & $0.45 \pm 0.10 \mathrm{ab}$ & $0.37 \pm 0.13 \mathrm{~b}$ & $0.29 \pm 0.10 \mathrm{c}$ \\
\hline Protonymph A & $\frac{1}{8}$ & $\begin{array}{c}2.23 \pm 0.34 \mathrm{~b} \\
1.91 \pm 0.40 \mathrm{ab}\end{array}$ & $1.70 \pm 0.14 \mathrm{~b}$ & $2.35 \pm 0.29 \mathrm{~b}$ & $1.77 \pm 0.19 \mathrm{~b}$ & $\therefore .72 \pm 0.22 \mathrm{c}$ & $0.89 \pm 0.16 \mathrm{~d}$ \\
\hline & +9 & $\begin{array}{l}1.91 \pm 0.40 \mathrm{ab} \\
0.71 \pm 0.20 \mathrm{~cd}\end{array}$ & $0.79 \pm 0.20 \mathrm{ab}$ & $1.92 \pm 0.13 \mathrm{c}$ & $1.00 \pm 0.15 \mathrm{a}$ & $1.16 \pm 0.25 \mathrm{~d}$ & $0.66 \pm 0.10 b c$ \\
\hline Q. & $\frac{\pi}{3}$ & $\frac{0.71 \pm 0.20 \mathrm{~cd}}{0.66 \pm 0.12 \mathrm{~b}}$ & $0.70 \pm 0.09 b$ & $0.80 \pm 0.09 \mathrm{bc}$ & $0.73 \pm 0.13 \mathrm{~b}$ & $0.64 \pm 0.18 \mathrm{~d}$ & $0.73 \pm 0.13 \mathrm{~b}$ \\
\hline & 오 & $\begin{array}{c}0.66 \pm 0.12 \mathrm{~b} \\
2.58 \pm 0.33 \mathrm{~b}\end{array}$ & $0.50 \pm 0.00 \mathrm{ab}$ & $0.50 \pm 0.16 \mathrm{c}$ & $0.40 \pm 0.10 \mathrm{abc}$ & $0.50 \pm 0.00 \mathrm{c}$ & $0.50 \pm 0.10 \mathrm{abc}$ \\
\hline Deutonymph A & $0^{1}$ & $\begin{array}{l}2.58 \pm 0.33 \mathrm{~b} \\
2.08 \pm 0.20 \mathrm{a}\end{array}$ & $1.60 \pm 0.19 \mathrm{ab}$ & $2.15 \pm 0.24 c$ & $1.75 \pm 0.30 \mathrm{ab}$ & $1.83 \pm 0.19 \mathrm{~d}$ & $1.58 \pm 0.16 \mathrm{c}$ \\
\hline & 우 & $\begin{array}{l}2.08 \pm 0.20 \mathrm{a} \\
0.85 \pm 0.19 \mathrm{~b}\end{array}$ & $1.37 \pm 0.31 \mathrm{a}$ & $1.92 \pm 0.20 \mathrm{ab}$ & $0.66 \pm 0.13 \mathrm{c}$ & $1.66 \pm 0.13 \mathrm{~b}$ & $0.71 \pm 0.10 \mathrm{c}$ \\
\hline Q. & $\hat{0}^{2}$ & $\begin{array}{l}0.85 \pm 0.19 \mathrm{~b} \\
0.75 \pm 0.00 \mathrm{a}\end{array}$ & $0.45 \pm 0.09 a$ & $0.75 \pm 0.21 \mathrm{~b}$ & $0.79 \pm 0.09 \mathrm{a}$ & $0.70 \pm 0.29 \mathrm{~b}$ & $0.68 \pm 0.12 \mathrm{a}$ \\
\hline Total immatures & 우 & $\begin{array}{l}0.75 \pm 0.00 \mathrm{a} \\
9.80 \pm 0.89 \mathrm{~b}\end{array}$ & $0.37 \pm 0.13 \mathrm{a}$ & $0.63 \pm 0.20 \mathrm{bc}$ & $0.50 \pm 0.16 \mathrm{a}$ & $0.50 \pm 0.00 \mathrm{c}$ & $0.42 \pm 0.13 \mathrm{a}$ \\
\hline Total immatures & $0^{\pi}$ & $\frac{9.80 \pm 0.89 b}{7.93 \pm 0.54 a}$ & $6.98 \pm 0.41 \mathrm{~b}$ & $8.96 \pm 0.59 \mathrm{~b}$ & $7.38+0.40 \mathrm{ab}$ & $7.12 \pm 0.47 \mathrm{c}$ & $4.91 \pm 0.45 \mathrm{c}$ \\
\hline Life cycle & 오 & $\begin{array}{l}\frac{7.93 \pm 0.54 a}{14.36 \pm 1.10 b} \\
\end{array}$ & $4.74 \pm 0.37 \mathrm{a}$ & $6.80 \pm 0.50 \mathrm{bc}$ & $3.55 \pm 0.20 \mathrm{~b}$ & $5.77 \pm 0.57 \mathrm{~d}$ & $2.87 \pm 0.20 \mathrm{c}$ \\
\hline Life cycle & $\hat{\theta}$ & $\begin{array}{l}14.36 \pm 1.10 \mathrm{~b} \\
12.09 \pm 0.80 \mathrm{a}\end{array}$ & $\frac{10.16 \pm 0.48 \mathrm{a}}{7.61 \pm 0.40 \mathrm{a}}$ & $12.31 \pm 0.81 \mathrm{c}$ & $10.13 \pm 0.67 \mathrm{a}$ & $10.08 \pm 0.61 \mathrm{~d}$ & $6.93 \pm 0.63 \mathrm{~b}$ \\
\hline & 오 & $12.94 \pm 0.69 c$ & $\frac{7.61 \pm 0.40 \mathrm{a}}{10.22 \pm 0.87 \mathrm{~b}}$ & $9.70 \pm 0.53 \mathrm{c}$ & $6.38 \pm 0.26 \mathrm{~b}$ & $8.60 \pm 0.67 \mathrm{~d}$ & $4.00 \pm 0.18 \mathrm{c}$ \\
\hline Adult longevity & 8 & $9.54 \pm 1.03 \mathrm{a}$ & $\frac{10.22 \pm 0.87 \mathrm{~b}}{6.15 \pm 0.54 \mathrm{~b}}$ & $12.09 \pm 0.66 \mathrm{c}$ & $7.63 \pm 0.71 \mathrm{~d}$ & $14.86 \pm 1.61 \mathrm{a}$ & $12.49 \pm 0.50 \mathrm{a}$ \\
\hline Jifo snan & 오 & $27.30 \pm 1.79 b$ & $\frac{6.15 \pm 0.54 b}{20.38 \pm 1.35 b}$ & $8.16 \pm 0.68 \mathrm{~b}$ & $5.29 \pm 0.50 \mathrm{c}$ & $10.30 \pm 0.84 \mathrm{a}$ & $6.96 \pm 0.68 \mathrm{a}$ \\
\hline Life span & o & $21.63 \pm 1.83 \mathrm{~b}$ & $\frac{20.38 \pm 1.35 b}{13.76 \pm 0.94 b}$ & $24.40 \pm 1.47 \mathrm{~d}$ & $17.76 \pm 1.38 \mathrm{c}$ & $\frac{24.94 \pm 2.22 \mathrm{e}}{19.90 \pm 1.51 \mathrm{~d}}$ & $\frac{19.42 \pm 1.13 \mathrm{~d}}{10.96 \pm 0.86 \mathrm{~d}}$ \\
\hline
\end{tabular}

Table (2): Longevity and fecundity of $T$. cucurbitacearum adult female reared on six eggplant cultivars at two temperatures and $70 \pm 5 \%$ R.H.

\begin{tabular}{|c|c|c|c|c|c|c|c|}
\hline \multirow{2}{*}{$\begin{array}{l}\text { Eggplant } \\
\text { cultivars }\end{array}$} & \multirow{2}{*}{ Temp. } & \multicolumn{4}{|c|}{$\begin{array}{l}\text { Period in days } \\
\end{array}$} & \multicolumn{2}{|c|}{ Fecundity } \\
\hline & & Pre-Oviposition & Oviposition & Post-Oviposition & Generation & No. of eggs & Daily rate \\
\hline \multirow{2}{*}{ Taska } & $25^{\circ} \mathrm{C}$ & $2.75 \pm 0.35 \mathrm{a}$ & $9.90 \pm 1.44 \mathrm{a}$ & $2.21 \pm 0.33 \mathrm{a}$ & $12.83 \pm 0.59 \mathrm{e}$ & $70.70 \pm 22 \mathrm{a}$ & $7.08 \pm 2.58 \mathrm{a}$ \\
\hline & $35^{\circ} \mathrm{C}$ & $2.10 \pm 0.38 \mathrm{a}$ & $8.64 \pm 0.45 \mathrm{a}$ & $1.75 \pm 0.32 \mathrm{a}$ & $9.03 \pm 0.55 \mathrm{c}$ & $47.58 \pm 13.01 \mathrm{a}$ & $5.50 \pm 1.61 \mathrm{bc}$ \\
\hline \multirow{2}{*}{ Black king } & $25^{\circ} \mathrm{C}$ & $2.25 \pm 0.32 \mathrm{c}$ & $8.73 \pm 0.83 \mathrm{bc}$ & $1.96 \pm 0.45 \mathrm{ab}$ & $16.61 \pm 1.10 \mathrm{~b}$ & $35.75 \pm 5.36 \mathrm{~b}$ & $4.09 \pm 0.67 \mathrm{~b}$ \\
\hline & $35^{\circ} \mathrm{C}$ & $1.92 \pm 0.33 \mathrm{a}$ & $6.47 \pm 0.59 \mathrm{~b}$ & $1.83 \pm 0.27 \mathrm{a}$ & $12.08 \pm 0.47 \mathrm{ab}$ & $33.25 \pm 8.24 \mathrm{~b}$ & $5.13 \pm 1.39 \mathrm{~d}$ \\
\hline \multirow{2}{*}{ Baladi } & $25^{\circ} \mathrm{C}$ & $1.58 \pm 0.39 \mathrm{~d}$ & $5.41 \pm 0.69 \mathrm{~d}$ & $1.88 \pm 0.19 \mathrm{a}$ & $12.59 \pm 0.92 \mathrm{e}$ & $31.50 \pm 9.05 \mathrm{~b}$ & $5.82 \pm 1.74 \mathrm{a}$ \\
\hline & $35^{\circ} \mathrm{C}$ & $1.23 \pm 0.27 \mathrm{~b}$ & $3.98 \pm 0.85 \mathrm{~d}$ & $1.45 \pm 0.36 \mathrm{bc}$ & $8.20 \pm 0.74 \mathrm{c}$ & $33.15 \pm 11.47 \mathrm{~b}$ & $8.32 \pm 1.18 \mathrm{a}$ \\
\hline \multirow{2}{*}{$\begin{array}{l}\text { Black Stream } \\
\text { beity }\end{array}$} & $25^{\circ} \mathrm{C}$ & $2.45 \pm 0.35 \mathrm{~b}$ & $8.95 \pm 0.55 \mathrm{~b}$ & $2.02 \pm 0.37 \mathrm{a}$ & $15.99+0.80 \mathrm{c}$ & $45.58 \pm 12.04 \mathrm{~b}$ & $5.09 \pm 1.21 \mathrm{~b}$ \\
\hline & $35^{\circ} \mathrm{C}$ & $1.84 \pm 0.19 \mathrm{a}$ & $6.25 \pm 0.68 \mathrm{~b}$ & $1.35 \pm 0.40 \mathrm{c}$ & $12.06 \pm 1.50 \mathrm{ab}$ & $41.41 \pm 11.74 \mathrm{~b}$ & $6.62 \pm 1.45 \mathrm{~cd}$ \\
\hline \multirow{2}{*}{ Roma } & $25^{\circ} \mathrm{C}$ & $2.08 \pm 0.27 \mathrm{c}$ & $8.10 \pm 0.73 \mathrm{c}$ & $1.91 \pm 0.34 \mathrm{ab}$ & $14.39 \pm 0.85 \mathrm{~d}$ & $35.50 \pm 5.53 \mathrm{~b}$ & $4.38 \pm 0.72 b$ \\
\hline & $35^{\circ} \mathrm{C}$ & $1.45 \pm 0.17 \mathrm{~b}$ & $4.56 \pm 0.64 \mathrm{c}$ & $1.62 \pm 0.16 \mathrm{ab}$ & $11.58 \pm 0.76 \mathrm{~b}$ & $34.50 \pm 7.39 \mathrm{~b}$ & $7.56 \pm 1.07 \mathrm{ab}$ \\
\hline \multirow{2}{*}{ White } & $25^{\circ} \mathrm{C}$ & $1.52 \pm 0.29 \mathrm{~d}$ & $5.64 \pm 0.43 \mathrm{~d}$ & $1.63 \pm 0.27 \mathrm{~b}$ & $17.80 \pm 2.51 \mathrm{a}$ & $41.66 \pm 6.40 \mathrm{~b}$ & $7.38 \pm 1.01 \mathrm{a}$ \\
\hline & $35^{\circ} \mathrm{C}$ & $1.25 \pm 0.28 \mathrm{~b}$ & $3.68 \pm 0.48 \mathrm{~d}$ & $1.85 \pm 0.27 \mathrm{a}$ & $11.86 \pm 0.85 \mathrm{a}$ & $29.42 \pm 5.66 \mathrm{~b}$ & $7.99 \pm 1.46 \mathrm{a}$ \\
\hline
\end{tabular}

Numbers in each column followed by different letters are significantly different ( $P=0.05$; Duncan's Multiple Range Test 
immature males and females of $T$. cinnabarinus (Boisd.) were not significantly influenced by the eight strawberry varieties (Kazak and Kibritçi, 2008). These results were different than our findings, whereas the eggplant cultivars and temperature had significant differences on the total immature stages of $T$. cucurbitacearum, as it averaged $(11.72 \& 7.11)$ days for White at $25^{\circ} \mathrm{C}$ and ( $7.11 \& 4.91$ ) for Taska at $35^{\circ} \mathrm{C}$, respectively.

Hot and dry weather accelerates the life cycle of spider mites (Haile and Higley, 2003). Therefore, high temperature provided in the laboratory could be a reason for the shorter developmental time of $T$. cucurbitacearum as observed in the current investigation. Rearing the mites at $35^{\circ} \mathrm{C}$ shortened its developmental time and enabled its populations to grow dramatically fast than at $25^{\circ} \mathrm{C}$. The female life cycle being the shortest when reared on leaves of "Taska" cultivar (10.08 and 6.93 days) at 25 and $35^{\circ} \mathrm{C}$, respectively. On the other hand, the longest one was observed on leaves of "White" cultivar (16.28 and 10.61) at the previous temperature degrees, respectively. The obtained result of life cycles is similar to that obtained by (Van de Vire et al., 1972 and Abd El-Mohsin 2011). Male showed similar trend as female but with slightly shorter periods (Table 1). Laing (1969) found nearly similar developmental time for males and females (16.1 and 16.9 days), respectively which differed from what was reported here (Table 1). Again,Van de Vrie et al. (1972) emphasized the occurrence of the differences between males and females as to development rate. The influence of several factors to mites, temperature among others, may explain the differences encountered by the authors.

Elongated longevity at decreased temperatures has been observed in many tetranychid species (Boudreaux, 1963). This was also observed in $T$. cucurbitacearum (Sayed), whereas longevity prolonged to (14.86) days on "Taska" at $25^{\circ} \mathrm{C}$; than on the other cultivars. In contrast, it decreased to 8.79 days at the same temperature on "White", which was significartly longer than those reared at $35^{\circ} \mathrm{C}$ (Table 1). The mite adult longevities in this work were similar to those of Abd El-Mohsin (2011) who stated that, female longevity of $T$. urticae recorded the shortest period (7.63 days) when reared on leaves of watermelon cultivar "Aswan" at $30^{\circ} \mathrm{C}$, and the longest ( 11.44 days) at $25^{\circ} \mathrm{C}$. Chahine and Michelakis (1994) reported that, no difference was found in adult longevity when egg plant, tomato and beans were used as hosts, but fecundity was indeed affected by the host plant. This indicates that, the developmental cycle of $T$. cucurbitacearum is influenced by several factors.
The respective durations of pre-oviposition, oviposition and post-oviposition of $T$. cucurbitacearum on six eggplant cultivars are summarized in (Table 2). Slightly significant host plant and temperature effects were observed on the pre-oviposition and post-oviposition periods. The oviposition period was significantly influenced by host plant cultivars and insignificantly by temperature. Females on "Taska" cultivar had the longest oviposition period, which was significantly different from those on the rest cultivars, where it ranged between 9.9 and 8.64 days at 25 and $35^{\circ} \mathrm{C}$, respectively.

The eggs were laid by female individualy and the daily fecundity of $T$. cucurbitacearum are presented in table (2). The maximum number of eggs was deposited between the $2^{\text {nd }}$ and $5^{\text {th }}$ days of the oviposition period. This result coincided with those presented by Abd El-Mohsin (2011) who reported that daily egg production of $T$. urticae reached its peak on fifth day on watermelon "Aswan" cultivar at $30^{\circ} \mathrm{C}(7.74$ eggs/ $/$ day); egg production decreased gradually thereafter.

The high egg production per female of $T$. cucurbitacearum reached 70.70 and 47.58 eggs with a daily rate of 7.08 and $5.50 \mathrm{egg} /$ female at 25 and $35^{\circ} \mathrm{C}$, respectively on "Taska" cultivar followed by $45.58,41.66,35.75,35.5,31.5$ eggs on Black stream beity, White, Black king, Roma and Baladi cultivars at $25^{\circ} \mathrm{C}$, respectively (Table, 2 ).

However, similar result on the reproduction of $T$. cucurbitacearum was recorded by Kim et al. (2008) who pointed out that the number of eggs laid per female of $T$. urticae was 78.0 eggs at $37^{\circ} \mathrm{C}$ on eggplant leaves.

The study revealed shorter period of life cycle for T. cucurbitacearum at $35^{\circ} \mathrm{C}$ and $70 \%$ R.H. that enabled this species to undertake several generations per hot months in summer. These characteristics give it the power to increase rapidly to very high numbers on suitable hosts and destroy it.

Finally, it can be concluded that, "White" cultivar is one of the least favorable cultivar for mite production than others, as it prolonged the period of life cycle and giving the less egg production.

\section{REFERENCES}

Abd El-Mohsin, M. M. A. 2011. Ecology, biology and control of some mites associated with watermelon at Qaluobia governorate. M. Sc. Thesis, Fac. Agric., Cairo Univ., Egypt, 150 pp. 
Abdel-Shaheed, G. A.; Hammad, S. M. and El-Sawaf, S. K. 1971. The Host Preference of the Carmine Spider Mite Tetranychus cucurbitacearum Sayed (Acarina, Tetranychidae). Zeitschrift für Angewandte Entomologie, 69 (1-4): 398-402.

Attiah, H. H.; Hanna, M. A.; Risk, R. A. and El-Saadany, G. 1978. Species differences between Tetranychus cucurbitacearum (Say.) and $T$. arabicus At. in response to rearing humidity, Zeitschrift für Angewandte Entomologie, 85 (1-4): 37-42.

Boudreaux, H. B. 1963. Biological aspects of some phytophagous mites. Ann. Rev. Entomol., 8: 137$-154$.

Carey, J. R. and Bradley, J. W. 1982. Developmental rates, vital schedules, sex ratios, and life tables for Tetranychus urticae, T. turkestani and $T$. pacificus (Acarina: Tetranychidae) on cotton. Acarologia, 23: 333-345.

Chahine, H. and Michelakis, S. 1994. Bioecological control studies on Tetranychus urticae Koch (Acarina, Tetranychidae). Bulletin IOBC/WPRS, 17 (1): 121-124.

Farrag, A. M. I. 1975. Biological and ecological studies on family Tetranychidae. Ph. D. Thesis, Fac. Agric., Cairo Univ., Egypt. 152 pp.

Gomaa, E. A.; El-Enany, M. A.; Hassan, M. F. and Hassan, A. F. 1987. Biological response of the two common spider mites Tetranychus urticae Koch and T. cucurbitacearum (Sayed) to Zea mays and Sorghum vulgare. Bull. Fac. of Agric., Univ. of Cairo, 38 (1): 255-262.

Haile, F. J. and Higley, L. G. 2003. Changes in soybean gas-exchange after moisture stress and spider mite injury. Environ. Entomol., 32: 433-440.

Hassan, A. S. and Zarier, M. A. 1956. Biology of the red spider mite, Eotetranychus cucurbitacearum Sayed (Acarina: Tetranychidae). Bull. Soc. Entom. Egypte, 40: 301-320.
Hazen, A.; Gerson, U. and Tahori, A. S. 1973. Life history and life table of the carmine spider mite. Acarologia, 15: 414-440.

Kazak, C. and Kibritçi, C. 2008. Population parameters of Tetranychus cinnabarinus Boisduval (Prostigmata: Tetranychidae) on eight strawberry cultivars. Turk. J. Agric. For., 32:19-27.

Kim, J.; Lee, S. K.; Kim, J. M.; Kwon, Y. R. and Kim, T. H. 2008. Effect of temperature on development and life table parameters of Tetranychus urticae Koch (Acari: Tetranychide) reared on eggplants. Korean Journal of Applied Entomology, 47 (2): 163-168.

Laing, J. E. 1969. Life history and life table of Tetranychus urticae Koch. Acarologia, 11 (1): 32-42.

Mansour, F. A. and Karachi, Z. 1994. Resistance to carmine spider mite in watermelon. Phytoparasitica, 22(1):43-45.

Morishita, M. and Yano, S. 1996. Economic injury level of two spider mites, Tetranychus urticae Koch and T. kanzawai Kishida in watermelon. Proceedings of the Kansai Plant Protection Society, 38:17-22.

Quinn,G.P and Keough,M.J. 2002. Experimental design and data analysis for biologists. books. google. com. eg -537 pages.

Reddy, G.P.V. 2001. Comparative effectiveness of an integrated pest management system and other control tactics for managing the spider mite Tetranychus ludeni (Acari: Tetranychidae) on eggplant. Exp. Appl. Acarol., 25: 985-992.

Vrie, M. Van de, Mcmurtry, J. A. and Huffaker, C. B. 1972. Ecology of tetranychid mites and their enemies - a review - III: biology, ecology, and pest status, and host-plant relations of tetranychids. Hilgardia, 41 (13): 343-432.

Zhang, Z. Q. 2003. Mites of greenhouses: identification, biology and control. CABI Publishing, Wallingford, $244 \mathrm{pp}$. 